\title{
La notion de référentiel appliquée à la garde des jeunes enfants. Une comparaison France-Suède
}

\section{Anne-Marie Daune-Richard}

\section{Résumé}

En utilisant la notion de référentiel et en l'appliquant à la famille, et aux politiques de garde déjeunes enfants, l'auteur analyse la construction et la mise en œuvre des politiques publiques concernant l'accueil des jeunes enfants. Cette analyse met en relation des référentiels «globaux » et des référentiels qualifiés de "spécifiques ». Ceux-ci définissent à un moment donné, dans une société donnée, le rapport enfant/société et le rapport hommes/femmes. L'étude révèle des définitions et des modes d'articulation bien différents de ces référentiels en France et en Suède.

\section{Citer ce document / Cite this document :}

Daune-Richard Anne-Marie. La notion de référentiel appliquée à la garde des jeunes enfants. Une comparaison FranceSuède. In: Recherches et Prévisions, n56, juin 1999. pp. 33-46;

doi : 10.3406/caf.1999.1851

http://www.persee.fr/doc/caf_1149-1590_1999_num_56_1_1851

Document généré le 31/05/2016 


\title{
La notion de référentiel appliquée à la garde des jeunes enfants
}

\author{
Une comparaison France-Suède
}

\author{
Anne-Marie Daune-Richard *
}

En utilisant la notion de référentiel et en l'appliquant à la famille, et aux politiques de garde de jeunes enfants, $l$ 'auteur analyse la construction et la mise en ouvre des politiques publiques concernant l'accueil des jeunes enfants. Cette analyse met en relation des référentiels " globaux » et des référentiels qualifiés de "spécifiques". Ceux-ci définissent à un moment donné, dans une société donnée, le rapport enfant/société et le rapport hommes/femmes. L'étude révèle des définitions et des modes d'articulation bien différents de ces référentiels en France et en Suède.

\footnotetext{
* Laboratoire d'économie et sociologie du travail (Aix enProvence).
}

a garde des jeunes enfants, analysée comme politique publique, constitue un des éléments des politiques sociales dirigées vers la famille. Il faut toutefois souligner que cette conception est loin d'être universelle. D'un côté, au regard de l'histoire, ce n'est que dans un passé relativement récent que la garde des jeunes enfants tend à s'inscrire pleinement dans des politiques de soutien public aux familles. Pendant longtemps elle a relevé des politiques d'assistance et de lutte contre les effets de la pauvreté qui, obligeant les mères à chercher un emploi, les empêchaient d'assumer leurs fonctions de soins aux enfants. De l'autre, dans les pays industrialisés, le statut de la famille et des politiques familiales est extrêmement divers.

Dans l'ensemble européen, " peu de pays (la France, l'Allemagne et le Luxembourg seulement) reconnaissent explicitement l'importance de la famille en tant qu'institution et les responsabilités de l'Etat pourassurer sa protection. Leur engagement se traduit par des structures et des institutions chargées d'énoncer et d'ap- pliquer les décisions politiques». D'autres "s'engagent dans leur constitution à aider la famille, mais ne disposent pas des structures institutionnelles nécessaires à la mise en aeuvre des politiques familiales". Enfin, "quelques pays ne s'engagent pas vis-à-vis des familles ni dans leur constitution ni dans leurs institutions" (Hantrais et Letablier, 1996, p. 55). Le cas le plus connu est celui du RoyaumeUni, qui rejette l'idée même d'une intervention de l'Etat dans la vie privée des personnes et des familles. De fait, pour ce qui nous intéresse ici, le soutien public à la garde des jeunes enfants est particulièrement pauvre en terme d'offre de places et inexistant en terme de soutien financier aux familles (Moss, 1991 ; CE, 1996).

\section{Une fonction sociale définie par une politique publique}

Ainsi, la garde des jeunes enfants, en tant que politique familiale, est-elle profondément articulée à une représentation sociétale du rapport public-privé qui fait que, lors- 
que le respect du privé domine, elle est définie en " creux ». Cen'est pas pour autant qu'elle n'existe pas. Dans ce cas, la conception publique de la garde des jeunes enfants en attribue la responsabilité aux familles et, plus généralement, au privé, celui-ci pouvant d'ailleurs relever d'une combinaison famille-marché. Avec le développement de l'activité des mères se développent ainsi, au Royaume-Uni, des modes de garde privés qui créent de fortes discriminations entre les familles. Bien qu'elle ne soit ni instrumentée ni budgétisée, on a bien affaire ici à une politique publique en tant qu'elle définit publiquement qui doit assurer cette fonction sociale.

En tout état de cause, la famille, même lorsqu'elle n'est pas institutionnellement organisée comme objet de politique publique (par un secrétariat d'Etat à la famille par exemple), est institutionnalisée par la loi qui établit, à travers elle, les règles d'alliance et de filiation et les responsabilités de chacun et chacune à cet égard. En définissant les modalités d'articulation du privé au collectif, elle constitue une institution fondamentale de l'ordre social.

\section{Entre un système de sens et un ensemble de pratiques}

On examinera la garde des jeunes enfants en tant qu'elle relève d'une construction sociale de la réalité, d'un rapport entre un système de sens et un ensemble de pratiques. Cette conception de l'action sociale est au principe de plusieurs approches de la genèse et de la mise en ceuvre des politiques publiques, en particulier de celles en terme de "paradigme sociétal " Jenson, 1989 ; Jenson et Sineau, 1998 a ; Merrien, 1990) et celle en terme de "référentiel " (Jobert et Muller, 1987). "Le paradigme sociétal est entendu comme un réseau ramifié de normes, de règlements et de lois qui donnent leur signification aux multiples rapports sociaux dans la mesure où cet ensemble comporte une série de prémisses qui concernent une vision des rapports d'égalité et de hiérarchie, des relations entre les institutions et leurs rôles ", « il est propre à chaque pays et varie en fonction des périodes historiques... " Jenson et Sineau, 1998 a p. 10). Cette approche a servi de cadre d'analyse à une comparaison des modes d'accueil des jeunes enfants présentée dans l'ouvrage cité et dont les résultats nourrissent les réflexions développées dans cet article.

A travers la question de la garde des jeunes enfants, le propos est de faire travailler la notion de référentiel dans un domaine auquel, à notre connaissance, elle a été peu appliquée : celui de la famille. Deux questions, symétriques et imbriquées, constitueront notre fil conducteur: qu'est-ce que la notion de référentiel peut apporter de spécifiqueà l'analyse des politiques concernant la garde des jeunes enfants ? En quoi le champ de la garde des jeunes enfants interroge-t-il la mise en œuvre de l'idée de référentiel ?

\section{" Le référentiel d'une politique... un ensemble de normes prescriptives}

"Le référentiel d'une politique est constitué d'un ensemble de normes prescriptives qui donnent un sens à un programme politique en définissant des critères de choix et des modes de désignation des objectifs » (Muller, 1994, p. 43). Il faut ajouter qu'un aspect essentiel des propositions de Bruno Jobert et Pierre Muller est de distinguer un "référentiel global » - qui concerne l'ensemble de la société - et des "référentiels sectoriels", et de définir leur rapport comme une nécessaire mise en cohérence des seconds avec le premier. Comment repérer et définir les référentiels intervenant dans l'élaboration des politiques publiques concernant la garde des jeunes enfants?

La garde des jeunes enfants se rattache au champ des politiques familiales. Celui-ci, en France, correspond bien a priori à l'approche du "secteur" proposé par les auteurs : un appareil législatif, une structure administrative (généralement rattachée au ministère des Affaires sociales), un bras opérationnel où se rencontrent les partenaires sociaux (la Caisse nationale des alloca- 
tions familiales) et des médiateurs, porteparole des intérêts de la société civile au regard de la famille. Les débats entre ces différents acteurs arrêtent une définition à chaque fois provisoire et, en France, souvent conflictuelle $d$ 'un référentiel sectoriel concernant la place de la famille dans la société et le rôle de l'Etat à cet égard. Toutefois, ce n'est pas à l'ensemble de ce référentiel "famille " que nous nous intéressons ici, mais à sa composante "garde des jeunes enfants ". Ce domaine de l'action publique apparaît à la fois comme une composante du secteur " famille " puisque ses appareils opérationnels passent largement par ceux du secteur et, en même temps, comme une composante particulière du fait de son articulation avec des référentiels spécifiques.

\section{Répérer et définir les référentiels de l'action publique}

En nommant des référentiels spécifiques est abordé le problème rencontré pour repérer et définir les référentiels construisant l'action publique en matière de garde des jeunes enfants. Le modèle de B. Jobert et $P$. Muller met en relation à chaque fois un référentiel sectoriel (concernant l'agri- culture, l'aéronautique) et le référentiel global. Dans le secteur - ou sous-secteur garde des jeunes enfants, deux référentiels majeurs émergent, qui sont globaux en ce qu'ils traversent l'ensemble de la société (ils ne concernent pas un secteur au sens défini supra), mais ils ne le sont pas au sens de $B$. Jobert et $P$. Muller en ce qu'ils ne disent pas le tout d'une société donnée à un moment donné. Ces référentiels sont - au moins provisoirement - qualifiés de spécifiques. Il s'agit, d'une part, du référentiel qui définit les responsabilités respectives des parents, pères et mères, et de l'Etat au regard de l'éducation et du bien-être des enfants et, $d$ 'autre part, de celui qui définit la place respective des hommes et des femmes dans la société. Ces deux référentiels sont en fait articulés car ils touchent tous deux à la place de l'individu, y compris l'enfant, dans la relation public-privé.

\section{L'enfant, un bien public ?}

Le schéma montre que les modalités de prise en charge de la garde desjeunes enfants se définissent au regard de deux conceptions. La première concerne la place de l'enfant dans la société. L'enfant est-il un "bien public "? Sioui, en quoi ? Etcomment

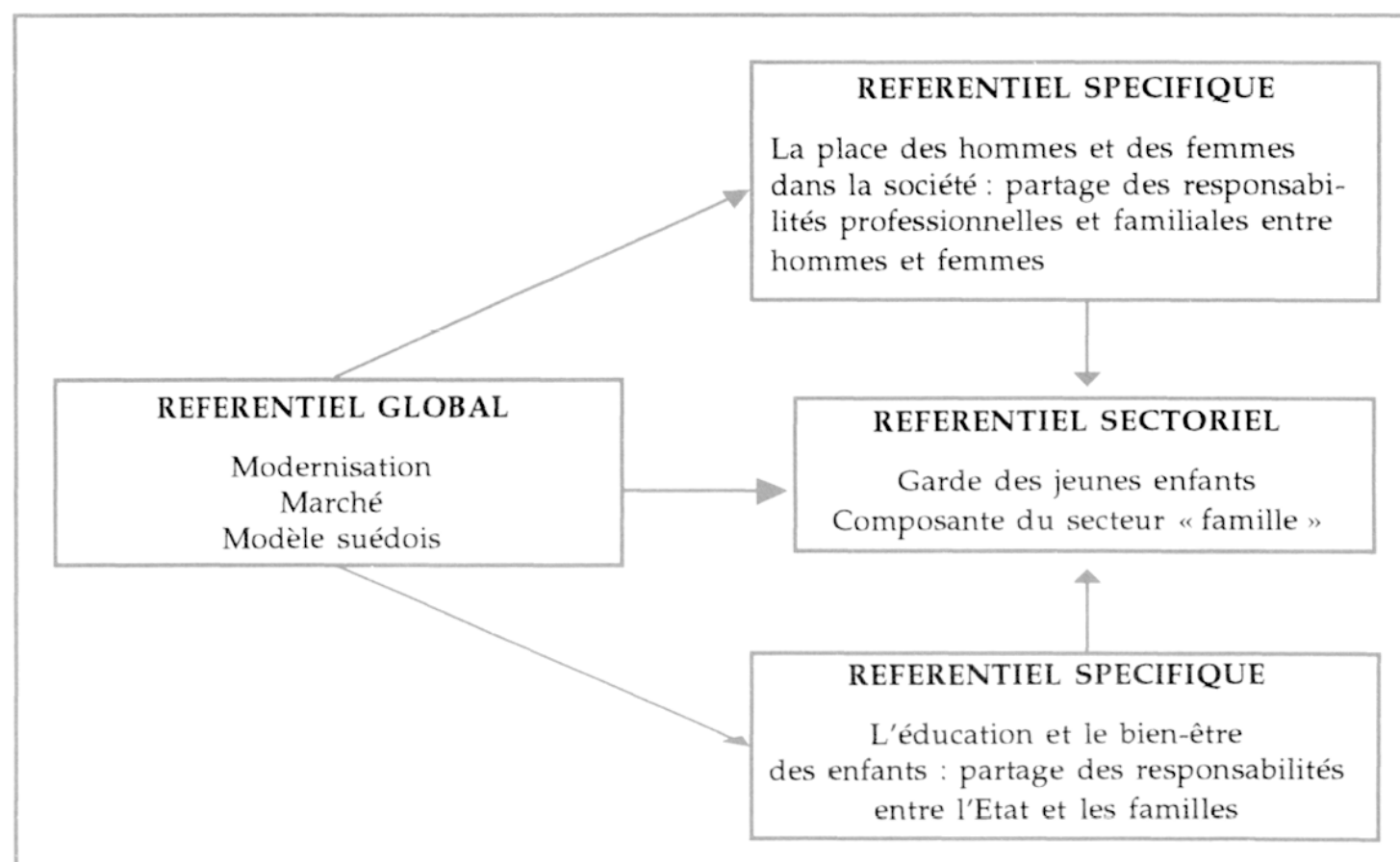


ce bien est-il géré ? Quelle relation s'établit entre les familles et l'Etat dans les responsabilités éducatives? Si l'on se réfère à l'exemple français, on peut considérer que le développement de l'intérêt public vis-àvis de l'enfant s'est articulé sur le principe d'égalité (donner les mêmes chancesà tous), mais aussi sur celui de la laïcité républicaine.

La création de l'école obligatoire (et gratuite) a été largement inspirée par la volonté de soustraire les enfants, futurs citoyens, à une instruction qui était aux mains de l'Eglise et à l'éducation de leurs mères tenues pour être sous influence religieuse. L'évolution des salles d'asile vers l'école maternelle s'inscrit elle aussi dans ce cadre : ce n'est pas un hasard si, en France, l'école maternelle est gratuite et rattachée à l'Education nationale. Ce n'est pas le cas dans la plupart des autres pays industrialisés où, jusqu'à l'âge de l'école obligatoire, l'accueil desjeunes enfants est onéreux et se fait dans des structures dont l'intitulé se rapproche le plus souvent de "centre de loisirs". En Suède, ce sont des « crèches " qui accueillent les enfants de 0 à 6 ans.

En France, tout se passe comme si l'Etat engageait totalement (gratuité) sa responsabilité éducative à partir de 3 ans, celleci étant, avant cet âge, l'affaire des familles (en 1993, seulement $23 \%$ des enfants de 0 à 3 ans sont gardés dans les services d'accueil collectifs financés par le secteur public ; CE, 1996 et Jenson et Sineau, 1998 a). C'est pourquoi il est difficile de considérer un secteur et un référentiel sectoriel. Les modalités de la garde des jeunes enfants répondent à des fins de soins, d'une part, à des fins éducatives, d'autre part, et sont partagées entre l'Education nationale (pour l'école maternelle), les Affaires sociales et les collectivités locales (pour les crèches) et les familles. Quant aux professionnels intervenant dans ce secteur, ils relèvent de statuts différenciés.

La deuxième conception est relative à la place respective des hommes et des femmes dans la société. P. Muller (1994, p. 43), évoquant la loi sur l'interruption volontaire de grossesse (IVG), met en relation son adoption avec "les modifications des représentations dominantes concernant la sexualité et la place des femmes dans la société ", qu'il considère implicitement comme des secteurs (ou, peut-être, comme un seul secteur; le propos est ambigu).

\section{Rapport hommes-femmes : des attentes sociales différenciées}

Certes, depuis les années soixante-dix, la question des femmes est institutionnalisée dans des appareils administratifs se référant d'abord à la condition féminine puis aux droits des femmes. Le gouvernement actuel avait, cependant, jugé bon de créer une Délégation interministérielle aux droits des femmes (récemment supprimée) actant ainsi les difficultés d'une politique sectorisée à cet égard. A notre sens, et plus fondamentalement, la question renvoie à l'impasse, relevée par Nicole-Claude Mathieu dès 1971 (réédité 1991), qui consiste à considérer le groupe des femmes comme un "en-soi " alors qu'il ne constitue qu'un élément du "système des sexes " présent dans toute société. C'est pourquoi nous considérons que le référentiel à prendre en compte est le rapport entre hommes et femmes qui génère des attentes sociales différenciées à leur égard.

La paternité et la maternité sont des constructions sociales articulées qui définissent la filiation et les responsabilités éducatives. Sans revenir aux thèses, controversées, décrivant "la découverte du sentiment de l'enfance" (Ariès, 1973) ou du sentiment maternel (Badinter, 1980), il est certain que l'autonomisation de l'enfant et du monde de l'enfance est, au regard de l'histoire, relativement récente, marquée par son institutionnalisation (école obligatoire au premier chef). Tant que les structures de production sont organisées dans le cadre familial, l'enfant est socialisé dans le cadre de la famille élargie.

Avec le salariat, les espaces de l'emploi et de la vie familiale se séparent et, avec l'urbanisation, la famille tend à se resserrer sur 
la conjugalité. L'homme devient le premier pourvoyeur des ressources du ménage et la femme se "spécialise " dans les activités domestiques et parentales. Les soins aux enfants deviennent une responsabilité maternelle, ceux-ci n'étant pas assurés lorsque la pauvreté les oblige à s'employer à $\mathrm{l}^{\prime}$ extérieur du foyer. Ce qui, au XIXe siècle, devient une question sociale entraînant, en France, la création des salles d'asile (Luc, 1997) et, en Suède, la prise en charge par un représentant de l'Etat du bien-être de l'enfant (Child Welfare Officer, Hwang et Broberg, 1992).

\section{Le modele familial repose sur la spécialisation des rôles}

Jusqu'à la deuxième moitié du XXe siècle, avec le développement du salariat, le modèle familial repose sur la spécialisation des rôles décrite par Talcott Parsons : aux femmes la responsabilité des soins aux enfants (qui détermine leurs engagements professionnels), aux hommes la charge des ressources financières de la famille. La question de la garde des jeunes enfants n'émerge dans le débat social que lorsque les femmes entrent massivement dans le salariat, sous l'effet conjugué de la transformation de la place des femmes dans la société et de l'appel de main-d'oeuvre par le système productifdans l'après-Seconde Guerre mondiale.

C'est donc une transformation du référentiel qui définit la place respective des hommes et des femmes tant dans le monde privé de la famille (abandon progressif du statut de mineure pour les femmes et de leur dépendance par rapport au mari) que dans l'espace public (accès à la citoyenneté civique) qui permet aux femmes d'accéder au statut $d$ 'individu à part entière et de ne plus être définies prioritairement comme mères et épouses. On assiste à un rapprochement des destins sociaux masculins et féminins.

Pour conclure ce premier point, le référentiel sectoriel " garde des jeunes enfants " et les deux référentiels spécifiques décrits semblent tout à fait articulés au référentiel global et à son évolution d'une définition en terme de modernisation à une définition en terme de marché telle que décrite par $B$. Jobert et P. Muller, mais avec des différences entre la France et la Suède.

\section{Dès son plus jeune âge, le petit Suédois est un citoyen}

En Suède, l'enfant est au coeur des préoccupations et des politiques familiales (Pitrou et Gaillard, 1989 ; Hantrais et Letablier, 1995 et 1996 ; Arve-Parès, 1996). L'enfant est, dès le plus jeune âge, un citoyen qui, même s'il n'est pas en mesure d'exercer des devoirs, a des droits : droit au bien-être, à êtreentouré et éduqué. La responsabilité des soins et de l'éducation est partagée, de façon complémentaire et différenciée, entre les parents et l'Etat mais les droits de l'enfant sont garantis par l'Etat. Ainsi la loi garantit-elle l'intégrité physique des enfants [les châtiments corporels sont strictement interdits, y compris de la part des parents (1)], le droit à être accueilli dans un service public de garde pour tout enfant de plus d'un an (âge en dessous duquel la grande majorité des enfants suédois est gardée par un parent en congé parental), dont les deux parents ont un emploi ou font des études, ainsi que la qualité du service offert.

En France, la famille définie par le Code Napoléon consacre, après la parenthèse révolutionnaire, un nouveau (2) modèle patriarcal qui rétablit l'autorité du chef de famille sur son épouse et ses enfants. La notion de "puissance paternelle " a sans doute profondément freiné l'émergence d'une considération des droits de l'enfant. Ceux-ci n'apparaissent en fait que dans une forme qui s'apparente plus à un partage de tutelle qu'à de réels droits, lorsque, à la fin du XIX ${ }^{e}$ siècle, l'enfant, en tant que futur citoyen, prend la figure d'un bien public: se dessinent alors des formes d'autonomisation de l'enfant par rapport à la famille et de prise en charge par l'Etat, soit en cas de défaillance de celle-ci (ainsi de la loi du 24 juillet 1889 instaurant la possibilité de la déchéance paternelle. Commaille, 1994, p. 156) soit pour le soustraire aux influences familiales (l'école de la République). 
Aujourd'hui ces principes inspirent encore largement le partage des responsabilités envers le jeune enfant : à l'Etat son éducation ainsi que sa protection lorsque les conditions familiales ou sociales lui sont défavorables ; aux familles la charge de l'amour et du bien-être. En deçà de l'âge scolaire (à 3 ans, la quasi-totalité des enfants français est scolarisée), la responsabilité des enfants repose largement sur les familles, les modes de garde extérieure à celles-ci ressortissant plus à une aide à la conciliation entre la vie professionnelle et la vie familiale qu'à l'intérêt propre à l'enfant.

Les différences relevées entre la Suède et la France au regard du rapport enfantsociété renvoient en fait à des conceptions différentes de la famille et de sa place dans la construction du lien social. La Suède développe un modèle de l'ordre social profondément ancré sur l'individu, ses droits et ses devoirs. La famille est ainsi conçue sur le modèle du contrat entre deux individus indépendants : elle est très peu institutionnalisée en tant que telle et ne fait pas, traditionnellement, l'objet d'une politique (Pauti, 1992). Ce qui ne veut pas dire qu'elle ne revête pas de l'importance aux yeux des Suédois (3), mais elle appartient pleinement à la sphère du privé et ce qui s'y passe ne regarde pas les affaires publiques, sauf sur le terrain de la protection de l'individu, y compris de l'enfant.

\section{En France, une vision de la famille comme institution}

En France, l'importance historique de la famille comme institution reste très prégnante. Emile Durkheim voyait en elle un "corps intermédiaire » entre l'Etat et les individus, indispensable au maintien de la cohésion sociale (Commaille, 1994, p. 149). En même temps, héritage de la Révolution, $l^{\prime}$ individu est une référence majeure. Ainsi, le cas français présente une tension entre une vision de la famille comme institution constitutive de l'ordre social et politique, et une conception où le contrat entre individus est central. A l'origine de l'idée de contrat, les débats ont plus concerné l'espace domestique (avec la question du mariage et du divorce) que l'espace civil du travail.

\section{Un accès tardif pour les femmes à l'espace public}

Le rapport hommes/femmes et sa place dans la construction du lien social s'exprime en particulier dans l'accès à l'espace public. Dans la construction des sociétés modernes, fondées théoriquement sur les droits et les devoirs individuels, cet accès a été - à des degrés divers - plus tardif pour les femmes que pour les hommes. Les femmes n'ont pas accédé à l'individualité et à la citoyenneté dans les mêmes termes et temporalités que les hommes.

La Suède et la France présentent à cet égard des différences importantes. D'un côté les Suédoises accèdent au droit de vote dans les années vingt, les Françaises en 1945. De l'autre, le Code Napoléon maintient les Françaises dans un statut de mineure jusque dans la deuxième moitié du $X X^{e}$ siècle (la notion de chef de famille ne disparaît qu'en 1970 et c'est en 1966 qu'une réforme des régimes matrimoniaux supprime la possibilité, pour un homme, de s'opposer à l'activité professionnelle de son épouse), alors que les Suédoises obtiennent des droits propres dans le mariage dès les années vingt.

Si en France, où domine la figure du "citoyen-producteur " masculin (Jenson, 1989), on peut repérer un lien entre l'exclusion des femmes du travail légitime et reconnu et leur exclusion de la citoyenneté civile et civique (Daune-Richard, 1997), tel ne semble pas être le cas en Suède. Les femmes y sont, plus encore que les Françaises, exclues du marché du travail (son accès est interdit aux femmes mariées jusqu'en 1938), mais beaucoup moins de la citoyenneté. Tout se passe comme si leurs fonctions de mère avaient fondé - en partie du moins - leur accès à la citoyenneté, et comme si l'homme, chef de famille, ne constituait pas - ou, en tout cas, moins qu'en France (4) - le para- 
digme du citoyen. Contrairement à ce qui s'est passé dans de nombreux pays, les Suédoises ont eu accès aux droits sociaux - troisième et dernière composante de la citoyennetéchez Thomas-Humphrey Marshall - directement en tant que citoyennes et mères et non en tant qu'ayants droit de leurs époux (Sainsbury, 1994).

Ainsi, la France et la Suède ne réagissentelles pas de la même façon à la crise démographique de l'entre-deux-guerres. Si la première développe des politiques de soutien à la fécondité et à la famille en protégeant la mère et, en particulier, dans la continuité des actions menées en ce sens depuis la fin du XIXe siècle, la mère salariée (Jenson, 1989), les Suédois développent une réflexion originale pour l'époque.

\section{Une vision * féministe- humaniste " de la femme modeme suédoise}

Dans un contexte où la famille et la fécondité ne sont pas des préoccupations politiques, deux économistes socio-démocrates, Alva et Gunnar Myrdal, publient en 1934 un livre - La crise démographique - qui lance un débat public sur la question. Analysant le problème, ils concluent que c'est en améliorant les conditions et le niveau de vie des familles en même temps que la condition des femmes (ils prônent des maternités volontaires et le droit au travail), qu'on soutiendra la natalité. Ainsi avancent-ils une vision " féministe-humaniste " de la femme moderne, économiquement indépendante (avec le soutien de l'Etat), combinant son rôle de mère et de citoyenne en participant pleinement à la vie civile et politique, qui inspirera largement l'évolution des politiques suédoises dans les décennies suivantes (Daune-Richard et Mahon, 1998).

Jusque dans les années cinquante, ce sont surtout les aides aux familles et aux mères qui se développent : allocations logements, allocations pour enfants à charge, gratuité des soins de maternité, allocations maternité pour toutes les mères, congés maternité pour les salariées. L'accès des femmes mariées au marché du travail n'est plus interdit, il n'est cependant pas favorisé (discrimination salariale instituée par des grilles de salaires séparées pour les hommes et pour les femmes, imposition conjointe du ménage, aucun développement des modes de garde publics pour les jeunes enfants).

\section{Pour chacun et chacune, travailler est un droit et une obligation}

Dans les années soixante et plus encore soixante-dix un modèle de "contrat entre les sexes " (Hirdman, 1994) où hommes et femmes seraient égaux devant l'emploi et les charges parentales est débattu et, finalement, retenu. Dans ce cadre, le développement des modes de garde est vu comme une condition de l'accès des femmes au marché et, ce faisant, de la mise en place d'un référentiel égalitaire. Référentiel dans lequel, pour chacun et chacune, travailler est un droit et une obligation, et prendre soin de ses enfants fait partie de ses droits et devoirs d'être humain. La mise en œuvre de ce référentiel introduit des réformes majeures dans la gestion du rapport entre sphères publique et privée : création d'un congé parental, s'adressant aux deux parents dès son origine (1974), et indemnisé en pourcentage du salaire antérieur (90\%) (5), développement de l'offre de garde publique pour les enfants (6), mesures en faveur de l'égalité salariale et mise en place de formes d'emplois à temps partiel réglementées et protégées. Les taux d'activité des femmes explosent, et en particulier ceux des mères de jeunes enfants (moins de 7 ans : $49 \%$ en 1970, $75 \%$ en 1980, $87 \%$ en 1989 ; Pauti, 1992).

Cependant, à la fin des années quatre-vingt, la mise en œuvre de ce référentiel égalitaire reste limitée: les congés parentaux sont massivement utilisés par les mères, l'emploi à temps partiel concerne principalement les femmes, et environ $40 \%$ d'entre elles y ont recours. Plus qu'un modèle " $a$ deux apporteurs égaux», c'est celui "à un apporteur trois quarts » qui est institué, mo- 
dèle où la femme reste, pour partie, économiquement dépendante du conjoint et de $l^{\prime}$ Etat. On peut toutefois penser à un modèle "de transition" (Anxo et Daune-Richard, 1991) car la part du temps partiel dans l'emploi féminin se réduit, le nombre d'heures travaillées augmente et la participation des pères aux congés parentaux grandit.

\section{La place sociale des Françaises : entre familialisme et féminisne}

En France, la place sociale des femmes est définie dans une tension entre deux pôles qui, selon Jacques Commaille (1993), traversent structurellement la société française : le "familialisme " comme "défense et promotion de l'institution familiale " et le "féminisme " comme reconnaissance de la femme en tant " qu' individu, sujet de droit ". Le premier pôle, héritier d'un modèle "fusionnel» de l'ordre familial, social et politique qui trouve ses racines dans l'Ancien Régime, se préoccupe de la fonctionnalité du familial par rapport au social et au politique (Commaille, 1994, p. 145 et suivantes). Il développe une vision holiste de la famille où chacun - et chacune - est assigné à une place dans un espace social fortement intégré UJ. Commaille se réfère à Louis Dumont, 1966 (réédité 1983)]. Le second pôle renvoie à une conception de la famille sur « le modèle du contrat » qui unit des individus par principe égaux en statut et en droit et suppose l'accès des femmes à l'individualité et à l'autonomie, au même titre que les hommes. De plus, comme tout contrat, il peut être dissous. Ce modèle de l'individuation, parce qu'il remet en cause l'existence de la famille en tant qu'institution fondamentale de l'ordre social, constitue, aux yeux des "familialistes", une menace pour la cohésion sociale.

Cette tension décrite par J. Commaille se manifeste par la coexistence des politiques et des institutions : secrétariat d'Etat à la famille et secrétariat d'Etat aux droits des femmes, par exemple. Dans le temps, l'un des pôles peut dominer, mais il ne fait pas disparaître l'autre. Ainsi, dès la fin du XIX ${ }^{c}$ siècle, les mères travailleuses ont-elles été protégées afin de préserver leur rôle familial vu comme prioritaire, mais pas interdites de travail salarié, comme dans d'autres pays (comme par exemple au RoyaumeUni avec la règle du marriage bar qui a perduré jusque dans les années cinquante).

\section{Encouragements à l'inactivité des mères}

Au cours des années quarante-cinqsoixante-dix, dans cette tension structurelle au sein du référentiel du rapport hommesfemmes, c'est le pôle "familialiste " qui domine (pour les développements ci-dessous, voir Jenson et Sineau, 1998 b). L'action publique concernant les enfants et la famille s'articule autour d'objectifs natalistes et d'encouragements à l'inactivité des mères (l'allocation de salaire unique apporte un complément de ressources aux familles où la mère est inactive). La garde des jeunes enfants est affirmée comme une spécialisation féminine (pas de développement des modes de garde collectifs).

Dans les années soixante-dix, le pôle individualiste prend le dessus et l'égalité entre hommes et femmes devient centrale dans le référentiel. La figure de la femme " libre " n'est plus celle qui «peut » rester au foyer parce que le ménage en a les moyens ou que ceux-ci sont complétés par l'Etat, mais celle qui a un métier. L'égalité des droits et des chances devient une question sociale et un enjeu politique (voir Jenson et Sineau, 1995). En 1974, sous la présidence de Valéry Giscard d'Estaing, est créé un Secrétariat à la condition féminine (qui sera élevé au rang de ministère à la fin de la décennie). C'est aussi pendant son septennat que sera votée la loi sur l'IVG et créé le congé parental d'éducation. Dans la même période, l'allocation de salaire unique, qui représentait une incitation à l'inactivité des mères, est supprimée et remplacée par une allocation pour frais de garde, en même temps que l'offre de garde publique se développe sensiblement.

Cependant, le familialisme reste prégnant. Contrairement à ce qui se passe en Suède 
à la même période, aucune réforme de la fiscalité des ménages n'est mise en œuvre : la taxation conjointe et le système du quotient familial sont toujours en vigueur aujourd'hui. Quant au congé parental, il n'est accessible au père que si la mère $y$ renonce et il n'est pas rémunéré.

\section{Idées de * libre choix " et de * conciliation $*$}

A partir du début des années quatre-vingt, $l$ 'articulation entre le référentiel concernant la garde des jeunes enfants et celui concernant les relations hommes/femmes se modifie. Si, dans la phase précédente, le soutien public à la garde des jeunes enfants est vu (malgré les ambivalences) comme un soutien à l'égalité hommes / femmes et à un nouveau statut de la femme dans la société, ce sont les idées de "libre choix" et de " conciliation entre vie familiale et vie professionnelle " qui fondent le nouveau référentiel. La garde des jeunes enfants est vue comme une forme "d'ajustement de la relation famille-emploi " (Hantrais et Letablier, 1995) dont les modalités relèvent des arbitrages individuels des familles, ce libre choix laissant de facto libre cours à la prégnance des rapports familiaux dans lequel l'autonomie économique tend à être une affaire $\mathrm{d}^{\prime}$ hommes et les responsabilités domestiques une affaire de femmes (7).

Ainsi, la responsabilité de l'ajustement incombe-t-elle préférentiellement aux femmes et mères et, de fait, on assiste dans les discours à un glissement sémantique des parents vers les mères, les pères n'ayant, pour leur part, jamais été réellement mis en avant (Jenson et Sineau, 1998 b), contrairement à ce qui s'est passé en Suède.

Dans l'approche de B. Jobert et P. Muller, le référentiel global renvoie à une représentation générale de la société. "Il est formé d'un ensemble de valeurs fondamentales qui constituent les croyances de base d'une société, ainsi qu'une série de normes qui permettent de choisir entre des conduites. (...) Le référentiel global n'est pas un consensus, mais il balise le champ intellectuel au sein duquel vont s'orga- niser les conflits sociaux" (Muller,1994, p. 147).

Dans le cas français, les fondateurs de cette approche voient deux référentiels globaux se succéder depuis la fin de la Seconde Guerre mondiale. L'un repose sur l'idée de "modernisation" et s'ancre dans la volonté de changement qui a marqué les années quarante-cinq - soixante-dix :le rejet du passé et la transformation-modernisation de la société en constitue les points cardinaux. L'autre naît dans les années quatre-vingt, dans la foulée de la crise économique : il affirme la primauté de l'entreprise et du marché, vante les mérites du " moins $d^{\prime} E$ tat " et se dirige vers l'intégration européenne.

\section{Le modèle suédois ébranlé dans ses fondements}

La Suède présente une périodisation et un espace de débat autour du référentiel global différents. D'un côté, le pays ayant, en 1940, choisi la neutralité, la guerre n'a pas constitué une rupture dans les valeurs qui fondent le référentiel suédois : mis en place dans les années trente, il a régi les relations sociales et professionnelles jusqu'à la fin des années soixante-dix. De l'autre, le fameux "modèle suédois " est construit autour de principes qui sont loin d'être au cœur des référentiels français: la négociation, la solidarité, l'égalité et l'emploi pour tous. Dans les années quatre-vingt et, plus encore, quatre-vingt-dix (Anxo, 1993), ce modèle est ébranlé dans ses fondements mêmes par une crise à plusieurs dimensions - économique, de l'emploi, de l'EtatProvidence - qui laisse un espace de développement pour un référentiel "marché " et des représentations plus individualistes des relations sociales. Cependant, les "valeurs " constitutives de ce modèle restent aujourd'hui des référents pour le débat social et politique.

Ces référentiels globaux interviennent-ils dans les évolutions du référentiel sectoriel (ou sous-sectoriel) "garde des jeunes enfants ", et si oui comment? Notre réponse 
sera clairement oui, et nous en montrerons les articulations (ou, au moins, quelquesunes d'entre elles), articulations qui transitent, à des degrés divers, par les référentiels "spécifiques " que nous avons repérés.

Dans la France des années soixante-dix, le référentiel égalitaire des rapports hommesfemmes s'articule au référentiel modernisateur de la société. A ce titre, il est un enjeu des programmes politiques qui oppose la droite et la gauche. Jane Jenson et Mariette Sineau (1995) montrent comment, dès le milieu des années soixante et tout au long des années soixante-dix, le candidat Mitterrand utilise le thème de la libération de la femme pour asseoir son image de candidat de la modernité et du changement, conduisant le Président Giscard d'Estaing à prendre des mesures (création d'un secrétariat $d^{\prime}$ Etat à la condition féminine, loi surl'IVG) prouvant sa modernitéà cet égard qui sont bien loin de faire l'unanimité dans son camp politique.

L'arrivée des socialistes au pouvoir, au début des années quatre-vingt, s'inscrit dans le cadre d'une crise de l'emploi qui ne cesse de grandir, tandis que les préoccupations démographiques, récurrentes en France, sont réactivées par la fin du baby-boom et la montée des pressions de l'extrême-droite sur ce terrain. L'essor du référentiel « marché " imprime sa marque à l'approche du rapport femmes/enfant/société.

\section{Des mesures, en France, pour ajuster famille et emploi}

La réduction des engagements de l'Etat et la flexibilité du travail sont à l'ordre du jour. D'un côté, l'accent est mis sur la diversification et l'individualisation des modes de garde au détriment du développement d'un grand service public: 300000 nouvelles places de crèches avaient été promises par le candidat Mitterrand, 70000 seront créées entre 1981 et 1991 Jenson et Sineau, 1995, pp. 242 et 247). De l'autre, des mesures sont prises pour flexibiliser et individualiser l'ajustement famille-emploi autour de la garde des enfants : allocations et/ou mesu- res fiscales destinées à aider les familles à recourir au marché (allocation de garde d'enfant à domicile, aide à la famille pour l'emploi d'une assistante maternelle agréée) ; incitations au développement des modalités temporelle, de l'ajustement (travail à temps partiel, congés parentaux).

On attend de ces mesures une réduction des budgets consacrés à la petite enfance (8) (les crèches étant décrites comme très onéreuses), mais aussi - et peut-être surtout - une diminution en volume de l'offre de travail des mères et des créations d'emplois de service. Enfin, ces mesures réactivent une vision renouvelée de la femme-mère : sa place première dans la famille est réaffirmée en ce que c'est sur elle que reposent les modalités de l'ajustement famille/emploi. Avec les notions de libre choix et de conciliation, le référentiel garde des jeunes enfants s'inscrit bien dans le référentiel global marché (et emploi, car ces deux dimensions apparaissent profondément articulées).

\section{Le petit enfant : l'affaire des femmes et des mères}

En même temps, dans le rapport hommes/ femmes/enfant/société, le référentiel garde des jeunes enfants éloigne le pôle égalitaire des rapports hommes/femmes au profit du pôle familialiste, où le petit enfant est avant tout l'affaire des femmes et des mères, et renonceà un projet qui égaliserait les conditions d'éducation par le développement d'un grand service public de la petite enfance.

En Suède, l'essor des modes de garde publics pour les jeunes enfants est directement articulé à celui d'un référentiel égalitaire des rapports hommes/femmes qui se développe au croisement de préoccupations politiques (voir supra), démographiques (la natalité qui était remontée dans les années trente et quarante connaît une nouvelle chute dans les années cinquante) et de préoccupations concernant la pénurie de main$\mathrm{d}$ 'œuvre qui touche la Suède après la guerre et jusque dans les années quatre-vingt. 
Dans les années soixante, après avoir fait appel à l'immigration, le choix est fait de recourir aux ressources nationales en maind'œuvre: les femmes. Dans un contexte favorable à l'emploi, les propositions en faveur d'un modèle égalitaire des rapports hommes-femmes s'ancrent dans les référents du "modèle suédois": solidarité, égalité et emploi pour tous (et toutes). Les préocupations natalistes sont réinterprétées à travers le modèle « féministe-humaniste " proposé par les Myrdal dans les années trente.

Enfin, si le développement d'un grand service public de garde des jeunes enfants est conçu comme un appui à l'intégration des femmes dans les normes du pleinemploi et de l'égalité, il est aussi conçu, au regard des enfants, suivant les principes de solidarité et d'égalisation ainsi que de qualité du service public qu'impose l'Etat-providence, garant du bien-être individuel et collectif. La crise du " modèle suédois ", patente pour les économistes depuis le milieu des années quatre-vingt, mais qui se révèle, brutalement, aux yeux des Suédois avec l'explosion du chômage au début des années quatre-vingt-dix (9), fragilise considérablement cette construction.

Un premier effet est induit par la réduction des dépenses publiques qui, dans un premier temps, pour ce qui nous concerne, s'applique aux revenus de remplacement des congés légaux (maladie, formation, parentaux) et aux conditions d'encadrement des enfants (diminution des personnels), mais devrait, dans un deuxième temps, toucher l'emploi féminin pour qui le secteur public est le premier employeur. Mais les effets de la crise ajoutés aux transformations socio-politiques du pays (tertiarisation de l'emploi qui réduit l'assise du syndicalisme ouvrier, base de la sociale-démocratie suédoise) et à l'intégration dans l'espace néolibéral européen laissent une place pour l'affirmation de référents individualistes. Concernant notre objet, des mesures ont été prises au début des années quatre-vingtdix, pendant l'alternance de droite et supprimées par les sociodémocrates à leur retour aux affaires, en $1994:$ l'autorisation de créer des entreprises privées, à but lucratif, de garde des enfants ainsi que la création d'une allocation destinée au parent inactif ou actif à temps partiel qui garde un enfant à la maison.

\section{Résistance du modèle égalitaire et du modèle public suédois}

Cependant, jusqu'à aujourd'hui, on constate une forte résistance du modèle égalitaire de " contrat entre les sexes" et du modèle "public " de garde des jeunes enfants qui ont constitué un des enjeux des dernières législatives. Les indicateurs objectifs, jusqu'en 1995, montrent une évolution, certes ralentie mais continue dans ce sens : la part des femmes dans la population active est stable et elles sont moins touchées par le chômage que les hommes; les efforts des pouvoirs publics en faveur de la garde des jeunes enfants se sont maintenus en dépit des difficultés budgétaires (le nombre d'enfants accueillis est passé de 532000 à 694000 entre 1990 et 1994 ; Arve-Parès, 1996).

Enfin, c'est en 1995 qu'a été mise en œuvre la loi obligeant les municipalités à offrir une place de garde pour chaque enfant (à partir d'un an) dont les parents travaillent ou suivent une formation. Quant aux congés parentaux, s'ils restent massivement utilisés par les mères, les pères y ont recours de façon grandissante (Arve-Parès, 1996) et l'incitation dans ce sens a été poursuivie par la création, en 1993, d'un mois de congé parental "réservé " au père (non transférable à la mère), mesure prise sous une majorité de droite.

La garde des jeunes enfants apparaît, en France, directement percutée par le référentiel marché-emploi qui a " travaillé " les ambiguïtés d'un référentiel rapport hommes/femmes peu stabilisé. Dans les années quatre-vingt, contrairement à d'autres périodes, le pôle " familialiste " de ce rapport apparaît renforcé davantage par les préoccupations en terme d'emploi et l'essor continu de l'actvité féminine que par un réel débat de société sur ce sujet. Le soutien public au développement de nou- 
velles formes de garde pour les jeunes enfants, dominées par les critères d'individualisation et de privatisation, $s^{\prime}$ inscrit aussi dans ce cadre, tout en s'appuyant sur un référentiel rapport enfant-société qui tend à considérer la responsabilité du jeune enfant comme une affaire de famille et, in fine, de femmes.

En Suède, le référentiel marché se développe et le mouvement induit par la convergence européenne ne peut que le renforcer. Cependant, pour ce qui concerne la garde des jeunes enfants, il se heurte à des référentiels - que nous avons caractérisés de "spécifiques"- beaucoup plus forts et stabilisés qu'en France. D'un côté, les Suédois restent extrêmement attachés au service public et à sa qualité, et ce qui est considéré comme une dégradation actuelle - l'augmentation de la taille des groupes d'enfants dans les crèches - fait l'objet d'un vif mécontentement. La privatisation de la garde des jeunes enfants ne se développe que faiblement, reste très minoritaire (moins de $10 \%$ des enfants de moins de 6 ans) et concerne le plus souvent des formes encadrées et réglementées (coopératives de parents). De l'autre, le référentiel égalitaire des rapports hommes-femmes "résiste". Sa cohérence avec le modèle suédois de citoyenneté et de construction de l'ordre social, fondé sur l'individu et dans lequel la famille est peu institutionnalisée, est probablement une des composantes de son ancrage. Une autre tient sans doute à la forte présence des femmes dans les syndicats (10) et la vie politique (plus de $40 \%$ des parlementaires sont des femmes). Or celles-ci semblent très opposées au modèle néo-libéral qui leur paraît menaçant au regard tant de leur accès à l'emploi que du soutien au modèle familial égalitaire. Dans le débat politique, les femmes peuvent ainsi apparaître comme une force de renouveau pour la démocratie (Mahon, 1995).
(1) Et les Suédois de passage en France sont très choqués par les comportements des parents français à cet égard tels qu'ils se donnent à voir dans l'espace public.

(2) Nouveau parce que déconnecté de l'origine religieuse de l'autorité.

(3) Ainsi la social-démocratie suédoise est-elle souvent évoquée à travers la métaphore de la "maison du peuple " qui renvoie à l'image du bien-être du foyer (good home) et aux relations d'égalité, de coopération et de respect mutuel qui y unissent les individus.

(4) Pour Anne Verjus (1997), le citoyen défini implicitement par la Révolution est le pater familias. Sur les relations entre maternité et citoyenneté, voir Tahon, 1997.

(5) Et, pour ce faire, création d'une "assurance parentale ", distincte de la sécurité sociale et financée à $80 \%$ par les cotisations des employeurs, le reste par l'Etat.

(6) En $1970,8 \%$ des enfants de moins de 6 ans sont accueillis dans une structure de garde financée par les pouvoirs publics, $30 \%$ en 1980, $50 \%$ en 1994. Daune-Richard, 1998.

(7) La venue d'un enfant a un effet sexué sur la répartition des temps professionnels et domestiques au sein du couple. Les pères français augmentent leur temps de travail professionnel et diminuent leur temps de travail domestique tandis que les mères font l'inverse. En Suède, le temps de travail professionnel reste stable chez les pères tandis qu'il diminue chez les mères, mais pères et mères augmentent leur temps de travail domestique (Anxo et Flood, 1998).

(8) Il serait intéressant de faire un bilan financier " global " de ces choix politiques, car pour ce qui concerne, par exemple, l'ouverture du congé parental et de l'APE au deuxième enfant, elle a coûté beaucoup plus cher que prévu ayant, en particulier, été l'objet d'un effet d'aubaine par des mères en difficulté sur le marché du travail (Afsa, 1996).

(9) Le chômage concerne environ $2 \%$ à $3 \%$ des actifs tout au long des années quatre-vingt, et $7 \%$ à $8 \%$ au début des années quatre-vingt-dix. En 1995 , il touchait $10 \%$ des hommes actifs et $8 \%$ des actives.

(10) En Suède, la syndicalisation est très forte (autour de $80 \%$ ) et à peu près égale chez les hommes et chez les femmes. 


\section{Références bibliographiques}

Afsa C., L'activité féminine à l'épreuve de l'allocation parentale d'éducation, Recherches et prévisions, décembre 1996, $\mathrm{n}^{\circ} 46$.

Anxo D., Les années 90 ou la fin du modèle suédois?, in Gazier B. (sous la direction de ), Emploi, nouvelles donnes, Economica, 1993.

Anxo D. et Flood L., Patterns of Time Use in France and Sweden, in Persson and Jonung (éds), Women's Work and Wages, Routledge, London, 1998.

Anxo D. et Daune-Richard, A.-M., La place relative des hommes et des femmes sur le marché du travail. Une comparaison France-Suède, Travail et emploi, 1991, $\mathrm{n}^{\circ} 47$.

Ariès P., L'enfant et la vie familiale sous l'Ancien Régime, Le Seuil, 1973.

Arve-Parès B., Entre travail et vie familiale : le modèle suédois, Lien social et politiques - RIAC, 1996, $\mathrm{n}^{\circ} 36$.

Badinter E., L'amour en plus, Flammarion, 1980.

$C E$, «Une étude des services pour les jeunes enfants dans l'Union européenne ", rapport ronéoté, DGV, Bruxelles, 1996.

Commaille J., L'esprit sociologique des lois, PUF, 1994.

Commaille J., Les stratégies de femmes. Travail, famille et politique, La Découverte, 1993.

Daune-Richard A.-M., Travail et citoyenneté : un enjeu sexué hier et aujourd'hui, in Bouffartigue P. et Eckert H. (sous la direction de), Le travail à l'épreuve du salariat : à propos de la fin du travail, L'Harmattan, 1997.

Daune-Richard A.-M., La garde des jeunes enfants en Suède : un enjeu pour l'égalité entre les hommes et les femmes, Les Cahiers du GEDISST, L'Harmattan, 1998, $\mathrm{n}^{\circ} 22$.

Daune-Richard A.-M. et Mahon R., La Suède. Le modèle égalitaire en danger ?, in Jenson J. et Sineau M. (sous la direction de), Qui doit garder le jeune enfant? Modes d'accueil et travail des mères dans l'Europe en crise, L.G.D.J, Paris, 1998.

Dumont L., Essais sur l'individualisme (réédition), Seuil, collection Points, 1983.

Hantrais L. et Letablier M.-T., La relation famille-emploi. Une comparaison des modes d'ajustement en Europe, Centre d'Etudes de l'Emploi, dossier 6, Nouvelle série, 1995.

Hantrais L. et Letablier M.-T., Famille, travail et politiques familiales en Europe, Cahiers du centre d'Etudes de l'Emploi, PUF, 1996.

Hirdman Y., Women : from Possibility to Problem ? Gender Conflict in the Welfare State. The Swedish Model. Research report, 3, Arbetlivcentrum, Stockholm, 1994.

Hwang C.P. et Broberg A., The historical and Social Context of Child Care in Sweden, in Lamb M.-E., Sternberg K. J. et Hwang C.P. et Broberg A., Child Care in Context, LEA, 1992. 
Jenson J., Paradigms and Political Discourse : Protective Legislation in France and the United States before 1914, Canadian Journal of Political Science, XXII, 1989, 2.

Jenson J. et Sineau M., Mitterrand et les Françaises. Un rendez-vous manqué, Presses de la Fondation nationales des Sciences Politiques, Paris, 1995.

Jenson J. et Sineau M. (sous la direction de), Qui doit garder le jeune enfant ? Modes d'accueil et travail des mères dans l'Europe en crise, L.G.D.J., Paris, 1998 a.

Jenson J. et Sineau M., La France. Quand "liberté de choix " ne rime pas avec égalité républicaine, in Jenson J. et Sineau M. (sous la direction de), Qui doit garder le jeune enfant? Modes d'accueil et travail des mères dans l'Europe en crise, L.G.D.J., Paris, 1998 b.

Jobert B. et Muller P., L'Etat en action. Politiques publiques et corporatismes, PUF, 1987.

Luc J.-N., L'invention du jeune enfant au XIXe siècle. De la salle d'asile à l'école maternelle, Belin, 1997.

Mahon R., "Swedish Unions in New Times : Women Workers as the Basis for Renewal ? ", communication à la rencontre annuelle de l'American Political Science Association, Chicago, 31 août-3 septembre 1995.

Mathieu N.-C., Notes pour une définition sociologique des catégories de sexe, in Mathieu, L'anatomie politique : catégorisation et idéologies du sexe, Indigo et Côté-femmes, 1991.

Merrien F.-X., Etat des politiques sociales : contribution à une théorie "neo-institutionnaliste ", Sociologie du Travail, 1990, $\mathrm{n}^{\circ} 3$.

Moss P., Day care for young children in the United-Kingdom, in Mehhuish E. C. and Moss P. (éds), Day care for young children. International perspectives, Tavistock/Routledge, Londres et New-York, 1991.

Muller P., Les politiques publiques, PUF, Que sais-je ?, 1994.

Pauti A., La politique familiale en Suède, Population, 1992, $\mathrm{n}^{\circ} 4$.

Pitrou A. et Gaillard A.-M., Familles de France et de Suède: à la recherche de nouveaux modèles, in Goudineau Y. (éd) Dynamiques familiales, Cahiers des Sciences Humaines, 1989, n 5 (3).

Sainsbury D. (1994), Women's and Men's Social Rights : Gendering Dimensions of Welfare State, in Sainsbury (éd.), Gendering Welfare States, Sage, Londres, 1994.

Tahon M.-B., La maternité comme opérateur de l'exclusion politique des femmes, in Tremblay M. et Andrew C. (sous la direction de), Femmes et représentation politique au Québec et au Canada, éditions du Remueménage, Montréal, 1997.

Verjus A., « Les femmes épouses et mères de citoyens ou de la famille comme catégorie politique dans la construction de la citoyenneté (1789-1848) ", thèse de doctorat d'Etudes Politiques, EHESS, Paris, 1997. 